\title{
Robotic versus Laparoscopic Intersphincteric Resection for Low Rectal Cancer: A Comparative Study of Short-term Outcomes
}

\author{
Jung Kyong Shin, M.D.', Yoonah Park, M.D. ', Hee Cheol Kim, M.D., Ph.D. ', Jung Wook Huh, M.D., Ph.D.', \\ Yong Beom Cho, M.D., Ph.D. ', Seong Hyeon Yun, M.D., Ph.D. ', Woo Yong Lee, M.D., Ph.D. ${ }^{,}$, Ho-Kyung Chun, M.D., Ph.D. ${ }^{2}$ \\ Department of Surgery, 'Samsung Medical Center, Sungkyunkwan University School of Medicine, ${ }^{2}$ Kangbuk Samsung Hospital, Sungkyunkwan University \\ School of Medicine, Seoul, Korea
}

Purpose: Intersphincteric resection (ISR) is a surgical option to preserve the anal sphincter for treatment of low rectal cancer. Laparoscopic ISR has been reported to be technically challenging. The Aim of this study was to assess the short-term outcomes of robotic ISR compared with a laparoscopic approach.

Methods: Ninety four consecutive patients who underwent laparoscopic $(n=60)$ or robotic $(n=34)$ ISR with hand-sewn coloanal anastomosis for low rectal cancer from January 2011 to December 2014 were included. Patient demographics, operative data, and histopathologic and postoperative outcomes were analyzed.

Results: There were no differences in demographic data including tumor location, which was $2.5 \pm 0.7 \mathrm{~cm}$ from the anal verge in the laparoscopic group and $2.7 \pm 0.9 \mathrm{~cm}$ in the robotic group. Mean operation time was significantly longer in the robotic group compared with the laparoscopic group ( $278 \pm 65.3$ minutes versus $225 \pm 66.9, p<0.001)$. With respect to histopathologic outcomes, patients with circumferential resection margin (CRM) less than $2 \mathrm{~mm}$ were observed more frequently in the laparoscopic group than in the robotic group $(18.3 \%$ versus $5.9 \%, p=0.050)$. The rate of postoperative morbidity was lower in the robotic group than in the laparoscopic group (14.7\% versus $35.0 \%$, $p=0.035$ ). Patients in the robotic group showed a low Clavien-Dindo score more frequently than those in the laparoscopic group $(p=0.049)$.

Conclusion: Robotic ISR is a safe and feasible procedure associated with a lower rate of narrow CRM and postoperative morbidity in spite of a longer operation time, compared with the laparoscopic approach. Prospective clinical trials with larger numbers of cases evaluating long-term oncologic and functional outcomes are required.

Keywords: Low rectal cancer, Intersphincteric resection, Robotic surgery, Laparoscopic surgery

This is an Open Access article distributed under the terms of the Creative Commons Attribution Non-Commercial License (http:// creativecommons.org/licenses/by-nc/4.0/) which permits unrestricted non-commercial use, distribution, and reproduction in any medium, provided the original work is properly cited.
Received August 31, 2015

Revised October 21, 2015

Accepted October 29, 2015

Corresponding author

Yoonah Park

Department of Surgery, Samsung

Medical Center, Sungkyunkwan

University School of Medicine, 81

Irwon-ro, Gangnam-gu, Seoul

06351, Korea

Tel: +82-2-3410-1532

Fax: +82-2-3410-6980

E-mail: yapark74@gmail.com

\section{INTRODUCTION}

Abdominoperineal resection (APR) has been considered as a standard surgical treatment of low rectal cancer for several decades. Recently, however, owing to improvement of surgical technique, advancement of stapling devices, adoption of preoperative chemoradiotherapy and evidences supporting that $1 \mathrm{~cm}$ of distal resection margin is adequate to achieve oncological safety especially for irradiated rectal cancer, sphincter-preserving operation has been attempted even 
for very low rectal cancer. ${ }^{1}$ Intersphincteric resection (ISR) is a surgical procedure which includes resection of internal anal sphincter combined with total mesorectal excision by dissecting intersphincteric space from perineal approach. ${ }^{2}$ Since ISR was firstly described in 1994, several studies reported that this procedure could be justified as an alternative to APR for treatment of very low rectal cancer with achievement of oncologic radicality as well as sphincter preservation. ${ }^{3}$

As with laparoscopic colectomy for cancer, laparoscopic surgery for rectal cancer has been evaluated in randomized clinical trials. ${ }^{4-8}$ They demonstrated that it provides favorable postoperative short-term outcomes and comparable oncologic results in comparison with open technique. In addition, several reports showed that laparoscopic proctectomy is associated with better voiding and sexual function than open approach in postoperative period. ${ }^{9,10}$ However, rectal cancer surgery with laparoscopic technique has not been widely spread in clinical practice as much as laparoscopic colectomy because it is technically demanding to carry out total mesorectal excision in narrow pelvic cavity with laparoscopic approach especially when the tumor is located just above or inside of the anal canal. As a result, application of surgical robot such as da Vinci $^{\circledR}$ Surgical System (Intuitive Surgical Inc., Sunnyvale, CA, USA) to rectal cancer surgery has expanded with expectation that a number of advantages of robotic systems, such as the stable operation field obtained by operator-controlled cameras, sustained and effective counter-traction when using a third robotic instrument, and endo-wrist functions of instruments, might mitigate the technical difficulties of laparoscopic total mesorectal excision. ${ }^{11}$

The aim of this study was to evaluate the safety and feasibility of robotic ISR for low rectal cancer by analyzing short-term outcomes in comparison with conventional laparoscopic approach.

\section{MATERIALS AND METHODS}

A total of 94 consecutive patients underwent laparoscopic $(n=60)$ or robotic $(n=34)$ ISR with hand-sewn coloanal anastomosis for primary low rectal cancer between January 2011 and December 2014 at Samsung Medical Center. Out of seven expert colorectal surgeons who carried out laparoscopic surgery, two surgeons (Park YA and Kim HC) conducted robotic surgery. They had previous experience of robotic surgery with approximate 200 and 10 cases, respectively. We used da Vinci S system for all of the cases. Data on patient demographics, intraoperative details, histopathologic outcomes, postoperative recovery and morbidity were collected from colorectal cancer database of our institution and chart review. Postoperative morbidity was graded according to the Clavien-
Dindo classification. ${ }^{12}$ Informed consent was obtained from all patients after we provided ample explanations of both surgical techniques in terms of the outline of surgery, currently available evidence for surgical outcomes, and cost.

Initial diagnostic work-ups for rectal cancer were composed of physical examinations including digital rectal examination, laboratory tests including serum level of carcinoembryonic antigen and carbohydrate antigen 19-9, colonoscopy with biopsy, chest X-ray, abdomino-pelvic computed tomography (CT) scan, chest CT scan and pelvic magnetic resonance images. Tumor location from the anal verge was measured by digital rectal examination and/or rigid sigmoidoscopy at the time of diagnosis and just before surgery. Rectal cancer was staged according to the tumor node metastasis (TNM) classification. Preoperative chemoradiotherapy (CRT) was considered for patients with T3-T4 tumors or node-positive disease. Radiotherapy was delivered to the whole pelvis at a dose of $45 \mathrm{~Gy}$ in 25 fractions for 5 weeks. The radiation field included the primary tumor and regional lymphatics including the mesorectum, presacral space, and the regional perirectal, internal iliac, presacral, and distal common iliac lymphatics. Chemotherapy consisted of a bolus injection of 5-fluorouracil, $500 \mathrm{mg} / \mathrm{m}^{2} /$ day for three days per cycle for the first and last weeks of RT or capecitabine, $850 \mathrm{mg} / \mathrm{m}^{2} /$ day for five days per week for five weeks. Surgery was carried out 6 to 8 weeks after the completion of preoperative CRT.

\section{Surgical technique}

For the laparoscopic procedures, a standard five-port system was applied as a usual manner. For the robotic procedures, a totally robotic approach with single docking technique $(n=30)$ or a hybrid approach $(n=4)$ was applied depending on the surgeon's preference. Surgical procedures of totally robotic approach were carried out as described in our previous report. $^{13}$ In brief, we used six-port system in which the camera port is placed on the midline of the $2 \sim 3 \mathrm{~cm}$ supra-umbilical area, two $8 \mathrm{~mm}$ trocars on the right lower abdomen, and each of the other three $8 \mathrm{~mm}$ trocars on the left mid-abdomen, epigastric and right suprapubic regions of the abdomen. The surgical cart was placed on the left caudal side of the surgical table in an oblique manner. This configuration is not changed during the whole procedure. After ligation of the inferior mesenteric vessels and mobilization of the leftsided colon including the splenic flexure was completed, the docking sites of two robotic arms are changed to be suitable for performing total mesorectal excision. Dissection was carried out all the way down to the pelvic floor and extended as far as possible to the intersphincteric space. Then, the robot was removed to perform the perineal procedure. 
When the mobilization of splenic flexure was considered to be imperfect, it was completed with laparoscopic technique before proceeding to the perineal procedure. The anus was closed with a purse string suture to prevent possible spillage of cancer cells during intersphincteric dissection. Intersphinteric dissection started from making circumferential incision between dentate line and intersphincteric groove according to the level of tumor. Circumferential dissection was extended in a cephalad direction to enter the previously created pelvic space. Specimens were extracted through the anus or minilaparotomy sites made by extension of the camera port depending on the bulk of the mesorectal fat or the surgeon's preference. In the hybrid approach, we applied a five-port system with a configuration similar to the totally robotic technique, but a $5 \mathrm{~mm}$ laparoscopic trocar was placed on the right upper abdomen instead of two $8 \mathrm{~mm}$ trocars on the epigastric and suprapubic area. Except that the ligation of inferior mesenteric vessels and colon mobilization were performed laparoscopically, the remaining procedures were not different from those of the totally robotic approach. Bowel reconstruction was conducted by hand-sewn coloanal anastomosis with or without colonic J-pouch. Protective ileostomies were created in selected patients.

\section{Statistical analysis}

Statistical analyses were carried out using the Statistical Package for the Social Sciences for Windows, version 22.0

Table 1. Patient demographics

\begin{tabular}{|c|c|c|c|c|}
\hline & $\begin{array}{l}\text { Total } \\
(\mathrm{N}=94)\end{array}$ & $\begin{array}{l}\text { Laparoscopic ISR } \\
(\mathrm{N}=60)\end{array}$ & $\begin{array}{l}\text { Robotic ISR } \\
(\mathrm{N}=34)\end{array}$ & $p$ value \\
\hline Age, years (range) & $56 \pm 11.3$ & $58 \pm 10.3(35 \sim 75)$ & $55 \pm 12.8(33 \sim 85)$ & 0.389 \\
\hline Gender & & & & 0.543 \\
\hline Male & $57(60.6)$ & $35(58.3)$ & $22(64.7)$ & \\
\hline Female & 37 (39.4) & $25(41.7)$ & $12(35.3)$ & \\
\hline Body mass index, $\mathrm{kg} / \mathrm{m}^{2}$ (range) & $23.3 \pm 3.9$ & $23.1 \pm 4.3(17.3 \sim 31.5)$ & $23.7 \pm 3.1(15.9 \sim 30.0)$ & 0.396 \\
\hline ASA score & & & & 0.310 \\
\hline 1 & $32(34.0)$ & $19(31.7)$ & $13(38.2)$ & \\
\hline 2 & $61(64.9)$ & $41(68.3)$ & $20(58.8)$ & \\
\hline 3 & $1(1.1)$ & $0(0.0)$ & $1(3.0)$ & \\
\hline Previous abdominal surgery & & & & 0.321 \\
\hline Yes & $22(23.4)$ & $16(26.7)$ & $6(17.6)$ & \\
\hline No & $72(76.6)$ & $44(73.3)$ & $28(82.4)$ & \\
\hline Preoperative CCRT & & & & 0.896 \\
\hline Yes & $63(67.0)$ & $40(66.7)$ & $23(67.6)$ & \\
\hline No & $31(33.0)$ & $20(33.3)$ & $11(32.4)$ & \\
\hline Preoperative CEA, ng/mL & $3.4 \pm 6.1$ & $2.9 \pm 3.8$ & $4.0 \pm 8.7$ & 0.053 \\
\hline Tumor location from AV, cm (range) & $2.6 \pm 0.8$ & $2.5 \pm 0.7(1.0 \sim 5.0)$ & $2.7 \pm 0.9(0.4 \sim 5.0)$ & 0.084 \\
\hline Clinical TNM stage & & & & 0.433 \\
\hline 0 & $1(1.1)$ & $1(1.7)$ & $0(0.0)$ & \\
\hline । & $33(35.1)$ & $21(35.0)$ & $12(35.3)$ & \\
\hline$\|$ & $9(9.6)$ & $5(8.3)$ & $4(11.8)$ & \\
\hline III & $46(48.9)$ & $28(46.7)$ & $18(52.9)$ & \\
\hline IV & $5(5.3)$ & $5(8.3)$ & $0(0.0)$ & \\
\hline
\end{tabular}

Values are number of patients $(\%)$ or mean \pm standard deviation. $I S R=$ intersphinteric resection; $A S A=$ American society of anesthesiologists; $C C R T=$ concurrent chemoradiotherapy; $C E A=$ carcinoembryonic antigen; $A V=$ anal verge. 
(SPSS, Chicago, IL, USA). The significance of differences between groups was evaluated using the chi-square test or $\mathrm{t}$-test, as appropriate. $p$ values of 0.050 or less were considered statistically significant.

\section{RESULTS}

Patient demographics are listed in Table 1. No significant differences were identified in terms of age, gender, body mass index, American Society of Anesthesiologists (ASA) score, previous abdominal surgery, preoperative chemoradiotherapy, preoperative serum level of CEA, tumor location from anal verge, or TNM stage. Two thirds of all patients underwent preoperative CRT. The mean distance between lower edge of tumor and anal verge at the time of diagnosis was $2.6 \pm 0.8 \mathrm{~cm}$.

\section{Operative outcomes}

Operative outcomes are summarized in Table 2. Operation time was significantly longer in the robotic group than laparoscopic group $(278 \pm 65.3$ vs. $225 \pm 66.9, p<0.001)$. The site of specimen delivery (transanal versus transabdominal) and reconstruction of rectal reservoir were not significantly different, although colonic J-pouches were more frequently constructed in the laparoscopic group than robotic group (43.3 vs. $5.9 \%, p=0.082)$. There was no case of open conversion in either group. Protective ileostomy was constructed in $90 \%$ of patients in robotic group and in $85 \%$ of those in laparoscopic group. All protective ileostomies were repaired by an average of 7.6 months in the laparoscopic group and 6.8 months in the robotic group, respectively ( $p=0.494$ ).

\section{Histopathologic outcomes}

Table 3 demonstrates the histopathologic results. The two groups showed no significant differences with respect to cell type, histologic grade, pathologic $\mathrm{T}$ and $\mathrm{N}$ category, or tumor size.

The distal resection margin was $1.5 \mathrm{~cm}$ in both the laparoscopic and robotic groups $(p=0.854)$. However, patients with narrow circumferential resection margin (CRM) were more frequently observed in laparoscopic group than in robotic group ( $p=0.033$ ). When we categorized CRM with the cut-off value of $2 \mathrm{~mm}$, patients who had a tumor with less than $2 \mathrm{~mm}$ of CRM were more frequent in laparoscopic group than in robotic group $(p=0.050)$. There were no significant differences in number of retrieved lymph nodes or positive lymph nodes.

\section{Postoperative outcomes}

Table 4 shows the postoperative outcomes. There were no significant differences in parameters associated with postoperative recovery including days to first bowel movement,

Table 2. Operative outcomes

\begin{tabular}{|c|c|c|c|c|}
\hline & $\begin{array}{l}\text { Total } \\
\text { (N=94) }\end{array}$ & $\begin{array}{l}\text { Laparoscopic ISR } \\
(\mathrm{N}=60)\end{array}$ & $\begin{array}{l}\text { Robotic ISR } \\
\quad(N=34)\end{array}$ & $p$ value \\
\hline Operation time, minutes & $244 \pm 70.1$ & $225 \pm 66.9$ & $278 \pm 65.3$ & $<0.001$ \\
\hline Site of specimen delivery & & & & 0.719 \\
\hline Transabdominal & $41(43.6)$ & $27(45.0)$ & $14(41.2)$ & \\
\hline Transanal & $53(56.4)$ & $33(55.0)$ & $20(58.8)$ & \\
\hline Reconstruction of rectal reservoir & & & & 0.082 \\
\hline Straight coloanal & 66 (70.2) & $34(56.7)$ & $32(94.1)$ & \\
\hline Colonic J-pouch & 28 (29.8) & $26(43.3)$ & $2(5.9)$ & \\
\hline Protective ileostomy & & & & 0.495 \\
\hline Yes & 83 (88.3) & $54(90.0)$ & $29(85.3)$ & \\
\hline No & $11(11.7)$ & $6(10.0)$ & $5(14.7)$ & \\
\hline Conversion to open surgery & $0(0.0)$ & $0(0.0)$ & $0(0.0)$ & 1.000 \\
\hline Estimated blood loss, mL & $174 \pm 108$ & $165 \pm 110$ & $190 \pm 104$ & 0.072 \\
\hline Perioperative transfusion & $1(1.1)$ & $1(1.7)$ & $0(0.0)$ & 0.449 \\
\hline Duration with ileostomy, months & $7.0 \pm 4.6$ & $7.6 \pm 4.6$ & $6.8 \pm 4.9$ & 0.494 \\
\hline
\end{tabular}

Values are number of patients $(\%)$ or mean \pm standard deviation. $I S R=$ intersphinteric resection. 
Table 3. Histopathologic outcomes

\begin{tabular}{|c|c|c|c|}
\hline & Laparoscopic ISR (N=60) & Robotic ISR (N=34) & $p$ value \\
\hline Cell type & & & 0.235 \\
\hline Adenocarcinoma & $58(96.7)$ & $33(97.1)$ & \\
\hline Mucinous carcinoma & $0(0.0)$ & $1(2.9)$ & \\
\hline Signet ring cell carcinoma & $2(3.3)$ & $0(0.0)$ & \\
\hline Histologic grade & & & 0.688 \\
\hline Well differentiated & $14(23.3)$ & $10(29.4)$ & \\
\hline Moderately differentiated & $42(70.1)$ & $23(67.7)$ & \\
\hline Poor differentiated & $2|3.3|$ & $0(0.0)$ & \\
\hline Undescribed & $2|3.3|$ & $1(2.9)$ & \\
\hline |y|pT category & & & 0.565 \\
\hline TO & $6(10.0)$ & $5(14.7)$ & \\
\hline Tis & $0(0.0)$ & $0(0.0)$ & \\
\hline $\mathrm{T} 1$ & $8(13.3)$ & $7(20.6)$ & \\
\hline $\mathrm{T} 2$ & $22(36.7)$ & $13(38.2)$ & \\
\hline Т3 & $22(36.7)$ & $9(26.5)$ & \\
\hline $\mathrm{T} 4$ & $2 \mid 3.3)$ & $0(0.0)$ & \\
\hline (y)pN category & & & 0.979 \\
\hline NO & $43(71.7)$ & $25(73.5)$ & \\
\hline N1 & $13(21.7)$ & $7(20.6)$ & \\
\hline N2 & $4(6.7)$ & $2(5.9)$ & \\
\hline Tumor size, cm & $2.7 \pm 1.4$ & $2.4 \pm 1.7$ & 0.457 \\
\hline Proximal resection margin, $\mathrm{cm}$ & $14.1 \pm 5.1$ & $16.8 \pm 6.7$ & 0.217 \\
\hline Distal resection margin, $\mathrm{cm}$ & $1.5 \pm 2.3$ & $1.5 \pm 0.9$ & 0.854 \\
\hline Circumferential resection margin, $\mathrm{mm}$ & & & 0.033 \\
\hline$\leq 1$ & $8(13.3)$ & $2(5.9)$ & \\
\hline $1 \sim 5$ & $18(30.0)$ & $7(20.6)$ & \\
\hline $5 \sim 10$ & $33(55.0)$ & $21(61.7)$ & \\
\hline$>10$ & $1(1.7)$ & $4(11.8)$ & \\
\hline Circumferential resection margin, $2 \mathrm{~mm}$ & & & 0.050 \\
\hline$<2$ & $11(18.3)$ & $2(5.9)$ & \\
\hline$\geq 2$ & $49(81.7)$ & $32(94.1)$ & \\
\hline Number of retrieved lymph nodes & $12.7 \pm 6.3$ & $12.1 \pm 5.5$ & 0.489 \\
\hline Number of positive lymph nodes & $1.3 \pm 5.0$ & $0.5 \pm 1.1$ & 0.641 \\
\hline
\end{tabular}

Values are number of patients $(\%)$ or mean \pm standard deviation. ISR $=$ intersphinteric resection.

days to normal diet, and length of hospital stay. However, rate of postoperative morbidity was significantly lower in the robotic group ( $14.7 \%$ vs. $35.0 \%, p=0.035)$. Rectovaginal fistula was observed in two patients in the laparoscopic group. Anastomotic leakage occurred in two patients in the laparoscopic group and three patients in the robotic group. One patient in the laparoscopic group and two patients in the robotic group had already undergone protective ileostomy, so 
Table 4. Postoperative outcomes

\begin{tabular}{lccc}
\hline & Laparoscopic ISR (N=60) & Robotic ISR $\left.\mathbf{N}^{*} \mathbf{N}=34\right)$ & $p$ value \\
\hline Days to the first bowel movement & $1.7 \pm 0.8$ & $1.6 \pm 0.9$ & 0.606 \\
\hline Days to normal diet & $2.2 \pm 0.8$ & $2.5 \pm 1.0$ & 0.136 \\
Length of hospital stay, days & $10.5 \pm 4.4$ & $10.3 \pm 3.4$ & 0.332 \\
\hline Morbidity & $21(35.0)$ & $5(14.7)$ & 0.035 \\
Wound complication & $1(1.7)$ & $1(2.9)$ & 0.681 \\
Anastomotic leak & $2(3.3)$ & $3(8.8)$ & 0.254 \\
Rectovaginal fistula & $2(3.3)$ & $0(0.0)$ & 0.282 \\
Intraabominal bleeding & $2(3.3)$ & $0(0.0)$ & 0.282 \\
Intraabodminal abscess & $2(3.3)$ & $0(0.0)$ & 0.282 \\
lleus & $4(6.7)$ & $0(0.0)$ & 0.124 \\
Urinary retention & $5(8.3)$ & $1(2.9)$ & 0.304 \\
Rectal mucosal prolapse & $2(3.3)$ & $0(0.0)$ & 0.282 \\
Pulmonary complication & $1(1.7)$ & $0(0.0)$ & 0.449 \\
Clavien-Dindo score & & & 0.049 \\
0 & $39(65.0)$ & $29(85.3)$ & 0.023 \\
1 & $0(0.0)$ & $0(0.0)$ & 1.000 \\
2 & $12(20.0)$ & $2(5.9)$ & 0.036 \\
$3 a$ & $2(3.3)$ & $0(0.0)$ & 0.452 \\
$3 b$ & $6(10.0)$ & $3(8.8)$ & 0.853 \\
4 & $1(1.7)$ & $0(0.0)$ & 0.452 \\
Reoperation & $5(8.3)$ & $1(2.9)$ & 0.304 \\
Mortality & $0(0.0)$ & $0(0.0)$ & 1.000 \\
\hline
\end{tabular}

Values are number of patients $(\%)$ or mean \pm standard deviation. $I S R=$ intersphinteric resection.

they were managed conservatively. However, the remaining one patient in each group underwent laparoscopic irrigation of peritoneal cavity with diverting loop-ileostomy. There were also significant differences in Clavien-Dindo scores between groups, and more severe postoperative complications occurred more frequently in the laparoscopic group than in the robotic group $(p=0.049)$. There were 5 cases of reoperation in the laparoscopic group and 1 case in the robotic group, due to anastomotic leakage, rectovaginal fistula, and rectal mucosal prolapse.

\section{DISCUSSION}

The current study compared the short-term outcomes of robotic and laparoscopic ISR for low rectal cancer. Robotic ISR showed longer operation time than laparoscopic approach. However, robotic approach was associated with lower rate of narrow CRM and postoperative morbidity. In most previous studies, it has been reported that robotic surgery for rectal cancer is associated with longer operation time than conventional laparoscopic approach. ${ }^{10}$ The difference ranges from 30 minutes to 90 minutes. In our study, operation time for robotic group was 50 minutes longer than laparoscopic group. It might be attributed to the following factors; (1) the additional time is needed for setting up such systems, (2) da Vinci S system is not perfect for multiple quadrant surgery. We needed to spend time to overcome collisions of robotic arms especially for the mobilization of splenic flexure colon, (3) because two surgeons who were involved in robotic surgery prefer to full mobilization of the splenic flexure colon especially for irradiated rectal cancer, whereas some of the other surgeons do not mobilize it for routine. As a result, the extent of surgical field might be wider in the robotic group, (4) one of the two robotic surgeons was a beginner with only 
10 cases of prior experience with robotic surgery, whereas all laparoscopic surgeons were expert surgeons with a huge amount of laparoscopic colorectal surgery. Because our study included the patients who were operated in the learning period of the beginning robotic surgeon, it might affect the longer operation time in robotic group. In previous studies, analyzing the operation time spent for each segment of procedures in totally robotic approach for rectal cancer, it was demonstrated that the step of procedure which show huge variation in time required for, was not rectal dissection but colonic mobilization. ${ }^{14}$ It means that colonic mobilization is the timelimiting step and there is room for reducing the operation time in this step. In other studies evaluating robotic rectal surgery with hybrid technique comparing with conventional laparoscopic surgery, the difference of operation time was less than that of totally robotic approach. ${ }^{10,15}$ Similarly, this finding may be interpreted that colon mobilization with robotic approach contributes to prolong the operation time. If the new generation of surgical robot can be developed suitable for multiple quadrant surgery without limitation of motions, it would be helpful to speed up robotic surgery for rectal cancer.

Circumferential resection margin of the resected specimen has been known to be a significant prognostic factor for patients with rectal cancer. ${ }^{16,17}$ Positive CRM is defined as involvement of circumferential margin with cancer cells or less than $1 \mathrm{~mm}$ of CRM. It has been reported that positive CRM is associated with increased rate of local recurrence as well as distant metastasis. ${ }^{18-20}$ In several studies, it was suggested that narrow CRM less than $2 \mathrm{~mm}$ can be predictive factor for local recurrence. ${ }^{21,22}$ In the present study, we demonstrated significant differences in CRM between the groups. When we categorized CRM at the level of $1 \mathrm{~mm}$, $5 \mathrm{~mm}$, and $10 \mathrm{~mm}$, patients of laparoscopic group showed narrow CRM (less than $1 \mathrm{~mm}$ or between 1 and $5 \mathrm{~mm}$ ) more frequently than those of robotic group. Similar result was identified with the cut-off value of $2 \mathrm{~mm}$. In laparoscopic ISR patients, 11 patients had CRM less than $2 \mathrm{~mm}$ (18.3\%), while 2 patients (5.9\%) had less than $2 \mathrm{~mm}$ margins in the robotic ISR group $(p=0.050)$. CRM is determined by the depth of mesorectal tumor spread and the quality of total mesorectal excision (TME). If the tumor invaded deep in the mesorectum or quality of TME is incomplete, the possibility of positive or narrow CRM is increased. Considering that clinical stage was not different between groups in our study, we can assume that the differences of CRM in two groups may be associated with different surgical approach. Surgical robot provides stable three-dimensional image which enhances improved visualization in the deep pelvic cavity and facilitate sharp dissection around mesorectal fascia and its extension into the intersphincteric space from abdominal cavity. This advantage might be attributed to the favorable result with respect to CRM in robotic group. In addition, when we look at the pathologic T category, T3-4 tumors are more frequently observed in laparoscopic group even though it was not statistically significant. It could be another explanatory reason for the different status of CRM.

In terms of postoperative outcomes, there was significantly lower overall morbidity in the robotic group compared to the laparoscopic group (14.7 vs. $35.0 \%, p=0.035$ ). When we focus on the major surgical complications such as anastomotic leak or rectovaginal fistula, significant difference was not observed between groups, in accordance with other studies. ${ }^{9,23}$ However, ileus, urinary retention, and rectal mucosal prolapse occurred relatively often in laparoscopic group compared to robotic group. Several studies demonstrated that robotic rectal surgery is associated with better voiding function especially in the immediately postoperative period. ${ }^{9}$ They explained that it might be come from better visualization provided by surgeon-controlled camera, 3-D image, and optimal countertraction with third robotic arm, and possibly resultant wellpreservation of pelvic autonomic nerves. Rectal mucosal prolapse is encountered occasionally following sphincterpreserving surgery for low rectal cancer. It may be related to the extent of resected internal sphincter, level of anastomosis and injury to anal sphincter muscle which may develop by anal retractor used during intersphincteric dissection from perineal side. Because intersphinteric dissection from abdominal side is facilitated with robotic approach owing to its advantages mentioned above, perineal intersphincteric dissection and the following damage to anal sphincteric can be minimized with robotic ISR. This might be one of the reasons why rectal mucosal prolapse was less frequent in robotic group than laparoscopic group.

The current study has several limitations. First, this is a single-center retrospective study. Inevitably, it has selection and information bias. Second, distribution of surgeons and the surgical expertise is different in each group. A total of seven surgeons were involved in laparoscopic surgery. Only two of them performed robotic surgery and one of the two robotic surgeons had little prior experience with robotic surgery. Third, we did not analyze long-term oncologic and functional outcomes. Therefore, in the future, we need to study TME quality, overall survival, recurrence free survival, local recurrence free survival, and anal sphincteric, voiding and sexual function in the randomized trial setting to evaluate robotic surgery for low rectal cancer.

In conclusion, robotic ISR is a feasible and safe approach for the treatment of low rectal cancer. Robotic ISR was associated with longer operation time than laparoscopic approach. The circumferential resection margin and postoperative overall 
morbidity were significantly better in patients treated by robotic ISR compared to those treated laparoscopically. Other short-term outcomes of robotic ISR were not inferior to those of laparoscopic resection. Further studies are required to evaluate long-term oncologic and functional outcomes in robotic ISR patients.

\section{REFERENCES}

1) Fischer A, Tarantino I, Warschkow R, Lange J, Zerz A, Hetzer $\mathrm{FH}$. Is sphincter preservation reasonable in all patients with rectal cancer? Int J Colorectal Dis 2010;25:425-432.

2) Schiessel R, Karner-Hanusch J, Herbst F, Teleky B, Wunderlich M. Intersphincteric resection for low rectal tumours. Br J Surg 1994;81:1376-1378.

3) Dahlberg M, Glimelius B, Pahlman L. Changing strategy for rectal cancer is associated with improved outcome. Br J Surg 1999;86:379-384.

4) Goh BK. Impact of laparoscopic resection for colorectal cancer on operative outcomes and survival. Ann Surg 2007;246:338-339; author reply 339-340.

5) Clinical Outcomes of Surgical Therapy Study G. A comparison of laparoscopically assisted and open colectomy for colon cancer. N Engl J Med 2004;350:2050-2059.

6) Guillou PJ, Quirke P, Thorpe H, et al. Short-term endpoints of conventional versus laparoscopic-assisted surgery in patients with colorectal cancer (MRC CLASICC trial): multicentre, randomised controlled trial. Lancet 2005;365:1718-1726.

7) Veldkamp R, Kuhry E, Hop WC, et al. Laparoscopic surgery versus open surgery for colon cancer: short-term outcomes of a randomised trial. Lancet Oncol 2005;6:477-484.

8) Lacy AM, Garcia-Valdecasas JC, Delgado S, et al. Laparoscopyassisted colectomy versus open colectomy for treatment of nonmetastatic colon cancer: a randomised trial. Lancet 2002;359:22242229.

9) Yoo BE, Cho JS, Shin JW, et al. Robotic versus laparoscopic intersphincteric resection for low rectal cancer: comparison of the operative, oncological, and functional outcomes. Ann Surg Oncol 2015;22:1219-1225.

10) Park SY, Choi GS, Park JS, Kim HJ, Ryuk JP. Short-term clinical outcome of robot-assisted intersphincteric resection for low rectal cancer: a retrospective comparison with conventional laparoscopy. Surg Endosc 2013;27:48-55.
11) Ballantyne GH. Robotic surgery, telerobotic surgery, telepresence, and telementoring. Review of early clinical results. Surg Endosc 2002;16:1389-1402.

12) Clavien PA, Barkun J, de Oliveira ML, et al. The Clavien-Dindo classification of surgical complications: five-year experience. Ann Surg 2009;250:187-196.

13) Park YA, Kim JM, Kim SA, et al. Totally robotic surgery for rectal cancer: from splenic flexure to pelvic floor in one setup. Surg Endosc 2010;24:715-720.

14) Bokhari MB, Patel CB, Ramos-Valadez DI, Ragupathi M, Haas EM. Learning curve for robotic-assisted laparoscopic colorectal surgery. Surg Endosc 2011;25:855-860.

15) Baik SH, Kwon HY, Kim JS, et al. Robotic versus laparoscopic low anterior resection of rectal cancer: short-term outcome of a prospective comparative study. Ann Surg Oncol 2009;16:1480-1487.

16) Wasserberg N, Gutman H. Resection margins in modern rectal cancer surgery. J Surg Oncol 2008;98:611-615.

17) Hermanek $P$, Junginger T. The circumferential resection margin in rectal carcinoma surgery. Tech Coloproctol 2005;9:193-199; discussion 199-200.

18) Quirke P, Durdey P, Dixon MF, Williams NS. Local recurrence of rectal adenocarcinoma due to inadequate surgical resection. Histopathological study of lateral tumour spread and surgical excision. Lancet 1986;2:996-999.

19) Park IJ, Kim JC. Adequate length of the distal resection margin in rectal cancer: from the oncological point of view. J Gastrointest Surg 2010;14:1331-1337.

20) Tilney HS, Rasheed S, Northover JM, Tekkis PP. The influence of circumferential resection margins on long-term outcomes following rectal cancer surgery. Dis Colon Rectum 2009;52:17231729.

21) Nagtegaal ID, van Krieken JH. The role of pathologists in the quality control of diagnosis and treatment of rectal cancer-an overview. Eur J Cancer 2002;38:964-972.

22) Nagtegaal ID, Marijnen CA, Kranenbarg EK, van de Velde CJ, van Krieken JH. Circumferential margin involvement is still an important predictor of local recurrence in rectal carcinoma: not one millimeter but two millimeters is the limit. Am J Surg Pathol 2002:26:350-357.

23) Park JS, Choi GS, Lim KH, Jang YS, Jun SH. Robotic-assisted versus laparoscopic surgery for low rectal cancer: case-matched analysis of short-term outcomes. Ann Surg Oncol 2010;17:31953202. 